\title{
Erreichbarkeitsmanagement in der betrieblichen Praxis
}

\author{
Daniel Grießhaber, Johannes Maucher, Uwe Laufs, \\ Katharina Staab, Zofia Saternus und Stephanie Weinhardt
}

\section{Zusammenfassung}

Smartphones und Laptops führen dazu, dass Arbeitnehmer über Handy oder E-Mail immer und überall arbeiten können und stets erreichbar sind. Da so die Grenzen von Privat- und Berufsleben immer mehr verschwimmen, befürchten Arbeitnehmervertreter und Politiker negative Folgen für Arbeitgeber und Beschäftigte. Deshalb suchen auch immer mehr Unternehmen nach Lösungen für ein effektives Erreichbarkeitsmanagement. Zur Umsetzung eines solchen Erreichbarkeitsmanagements können sowohl organisatorische als auch technische Ansätze verfolgt werden. Dieser Beitrag gibt Einblick in Ergebnisse des Forschungsprojekts SANDRA. Das Projekt entwickelt neben

D. Grießhaber $\cdot$ J. Maucher

Hochschule der Medien, Stuttgart, Deutschland

E-Mail: griesshaber@hdm-stuttgart.de; maucher@hdm-stuttgart.de

U. Laufs $(\triangle)$

Fraunhofer IAO, Stuttgart, Deutschland

E-Mail: uwe.laufs@iao.fraunhofer.de

K. Staab

Fachgebiet Marketing \& Personalmanagement, Technische Universität Darmstadt,

Darmstadt, Deutschland

E-Mail: katharina.staab@bwl.tu-darmstadt.de

Z. Saternus

Goethe Universität Frankfurt, Frankfurt am Main, Deutschland

E-Mail: saternus@wiwi.uni-frankfurt.de

S. Weinhardt

Fraunhofer IAO, Stuttgart, Deutschland

E-Mail: stephanie.weinhardt@iao.fraunhofer.de 
organisatorischen Lösungen auch einen Erreichbarkeits-Assistenten für Smartphones, der in Pilot-Unternehmen erprobt wird. Zudem zeigt der Beitrag die Erprobung und Evaluierung der Projektergebnisse in der betrieblichen Praxis auf.

\subsection{Motivation}

Die große Verbreitung moderner Informations- und Kommunikationstechnik (IKT), insbesondere des Internets und des Mobilfunks, prägen und verändern das Arbeitsumfeld grundlegend und nachhaltig (Sayah 2013; Tarafdar et al. 2007). Moderne IKT-Technologien steigern die Erreichbarkeit und die Möglichkeit zu arbeiten, unabhängig von Ort und Zeit im Sinne des ,,being connected 24/7“. Nicht nur Arbeitsleistung und Produktivitätsdruck erhöhen sich durch die Nutzung moderner IKT, ebenso wird die Trennung zwischen Beruf und Privatleben sowohl in zeitlicher als auch räumlicher Hinsicht zunehmend aufgehoben (David et al. 2014; Fonner and Stache 2012). Angesichts steigender Fehltage aufgrund psychisch verursachter Erkrankungen (Ayyagari et al. 2011) fürchten insbesondere Arbeitnehmervertreter und Politiker negative gesundheitliche Folgen für Beschäftigte aufgrund zunehmender Entgrenzung von Privat- und Berufsleben durch permanente technologische Erreichbarkeit. Es ist zu beobachten, dass eine steigende Anzahl an Unternehmen nach Lösungen im Sinne eines gesundheitsfördernden und effektiven Erreichbarkeitsmanagements für ihre Beschäftigten sucht. Aktuell reichen diese von Schulungen der Mitarbeitenden bis hin zu ,digitalen Sperrstunden“, z. B. durch das Abschalten des E-Mail-Servers nach Feierabend. Vielfach bieten diese Lösungen allerdings eher globale Lösungen, ohne die individuellen Bedürfnisse der Beschäftigten zu berücksichtigen. So deuten beispielsweise die Ergebnisse einer qualitativen Studie von Stock-Homburg et al. (Stock et al. 2014) darauf hin, dass für Führungskräfte bzgl. unterschiedlicher Lösungen im Bereich des Erreichbarkeitsmanagements größerer Bedarf besteht als für Mitarbeitende ohne Führungsverantwortung. Darüber hinaus spielt der Grad der Internationalisierung der Tätigkeit eine Rolle für die Gestaltung des Erreichbarkeitsmanagements. Das Forschungsprojekt SANDRA adressiert das betriebliche Erreichbarkeitsmanagement sowohl mit organisatorischen Maßnahmen als auch mit einer technischen Lösung, einem Erreichbarkeitsassistenten für Smartphones.

\subsection{Organisatorische Maßnahmen}

Neben einer speziell entwickelten Software sind organisatorische Maßnahmen das Mittel der Wahl, um das Erreichbarkeitsmanagement in einem Unternehmen zu verbessern. Hierbei sollten sowohl die Beschäftigten als auch Arbeitgeber und Führungskräfte gezielt angesprochen werden und praxisnahe Maßnahmen erhalten, die sich schnell und intuitiv durchführen lassen. Im Rahmen von SANDRA wurde basierend auf den Ergebnissen der 
empirischen Erhebungen (vgl. Kap. Beitrag 1) ein Maßnahmenkatalog entwickelt, der diese Sichtweisen adressiert. Die Beschäftigten erhalten dabei beispielsweise anschaulich aufgearbeitete Informationen zu dem Prinzip und der Notwendigkeit von Erreichbarkeitsmanagement und können ihr eigenes Erreichbarkeitsverhalten auf Basis einfacher Materialien reflektieren, während bei Führungskräften der Fokus auf ihrer Funktion als Vorbild und Organisator von Teams liegt. Durch eine Sammlung von ergänzenden Materialien und Tools sowie rechtlichen und organisatorischen Maßnahmen wurden auch für Arbeitgeber gezielte Hilfsmittel entwickelt, um das Erreichbarkeitsverhalten im Unternehmen zu hinterfragen und ggf. zu verbessern.

Bei der Entwicklung des Maßnahmenkatalogs wurden insbesondere drei Ziele verfolgt:

\section{1.) Schaffung eines stärkeren Bewusstseins für Erreichbarkeitsmanagement}

Ein erfolgreiches Erreichbarkeitsmanagement ist für die Gesundheit und das Wohlbefinden von Beschäftigten zentral und sollte daher überall dort, wo moderne Informations- und Kommunikationstechnologien für berufliche Tätigkeiten eingesetzt und auch außerhalb des Arbeitsplatzes verwendet werden, ein Thema sein. Gleichzeitig und Beschäftigten kaum eine Rolle spielt. Unsere Maßnahmen verfolgen daher das Ziel, das Bewusstsein für die Bedeutung von Erreichbarkeitsmanagement zu stärken.

2.) Steigerung der Kommunikation über Erreichbarkeit und Erreichbarkeitsverhalten Damit Erreichbarkeitsmanagement funktionieren kann, muss klar geregelt sein, unter welchen Umständen eine wie geartete Erreichbarkeit erwartet wird. Hier zeigen sich bei zahlreichen Mitarbeitern und Teams deutliche Defizite in der Kommunikation. Diese kommunikative Lücke soll durch unsere Maßnahmen geschlossen werden, um ein erfolgreiches Erreichbarkeitsmanagement zu ermöglichen.

3.) Förderung der Möglichkeit eines individuell passenden Erreichbarkeitsmanagements

Wie bereits in Kap. 3 geschildert, kann es nicht „,die eine“ Erreichbarkeitslösung geben, die für alle Beschäftigten ideal funktioniert. Ein zentrales Ziel unserer Maßnahmen ist es daher, die Ermöglichung solcher Erreichbarkeitslösungen zu fördern, die die individuellen Präferenzen des einzelnen Mitarbeiters berücksichtigen.

Im Folgenden wird eine Übersicht an Maßnahmen präsentiert, die auf Basis dieser Ziele entwickelt wurden.

\subsubsection{Beispielhafte Maßnahmen für Arbeitgeber}

Die Aufgabe des Arbeitgebers besteht darin, passende Voraussetzungen zu schaffen, die ein gesundes Erreichbarkeitsmanagement in seinem Unternehmen ermöglichen. Tab. 5.1 gibt eine Übersicht, an welchen Stellen Arbeitgeber ansetzen können, um das Erreichbarkeitsmanagement in seinem Unternehmen zu evaluieren und zu verbessern. 
Tab. 5.1 Maßnahmen für Arbeitgeber

\begin{tabular}{l}
\hline Titel der Maßnahme \\
\hline Überprüfung des \\
Erreichbarkeitsmanagements \\
im Unternehmen
\end{tabular}

Kurzerklärung der Maßnahme

Untersuchungen zeigen, dass viele Beschäftigte glauben, dass

von ihnen eine stärkere Erreichbarkeit erwartet wird, als dies

tatsächlich der Fall ist. Dies trifft insbesondere in Unternehmen

zu, in denen es wenig direkte Absprachen zur Erreichbarkeit

gibt. Auch bei anderen erreichbarkeitsbezogenen Themen

können sich die Wahrnehmungen unterscheiden. Überprüfen Sie

daher die Situation in Ihrem Unternehmen, zum Beispiel durch

eine anonyme Mitarbeiterbefragung.

Schaffen von Standards für $\quad$ Schaffen Sie Standards für Erreichbarkeitsmanagements. Legen

Erreichbarkeitsmanagement Sie dazu Zeiten fest, in denen eine Erreichbarkeit erwartet wird, im Unternehmen

und Zeiten, in denen keine oder nur eine eingeschränkte

Erreichbarkeit gilt. Definieren Sie zulässige Ausnahmen von

diesen Regeln, wie individuelle Wünsche oder klar

festzulegende Notfälle. Halten Sie diese Standards schriftlich

fest, z. B. im Rahmen Ihrer Betriebsvereinbarung, und sorgen

Sie dafür, dass Ihre Beschäftigten über diese Standards

Bescheid wissen.

\begin{tabular}{l|l}
\hline Fördern individueller & Die Forschung zeigt, dass Beschäftigte unterschiedliche
\end{tabular}

Lösungen

Bedürfnisse und Erwartungen haben, wie genau sie arbeiten

wollen. Dies gilt auch für die Verknüpfung von Arbeit- und

Privatleben, die in einem unterschiedlichen Ausmaß gewünscht

wird. Respektieren Sie diese Wünsche und regen Sie über Ihre

Erreichbarkeitsstandards hinaus individuelle Vereinbarungen

innerhalb Ihrer Teams und Arbeitsgruppen an. Stellen Sie

hierfür beispielsweise Leitfäden zur Verfügung und sorgen Sie

dafür, dass die Vereinbarungen schriftlich festgehalten werden.

Sehen Sie von starren Pauschallösungen ab: Diese können den

individuellen Bedürfnissen der Beschäftigten und ihrer

Situation kaum gerecht werden und führen oft dazu, dass die

Chancen der flexibleren Arbeitswelt nicht ausgenutzt werden

können.

\begin{tabular}{l|l}
\hline Unterstützung der & Ihre Maßnahmen sind nur dann wirksam, wenn Ihre
\end{tabular}

Beschäftigten durch $\quad$ Beschäftigten ihre Bedeutung verstehen. Motivieren Sie Ihre

passendes

Informationsmaterial

Beschäftigten dazu, sich mit Erreichbarkeitsmanagement

auseinandersetzen. Bieten Sie Ihnen dafür beispielsweise

kostenlose Broschüren oder niedrigschwellige Workshops an,

oder schulen Sie sie in der Nutzung von

Erreichbarkeitsanwendungen.

Schaffung eines langfristig

wirksamen

Eine gute Maßnahme sollte regelmäßig hinterfragt und

Erreichbarkeitsmanagements

überarbeitet werden. Führen Sie daher regelmäßig Evaluationen

in Form von Befragungen durch. Geben Sie den Beschäftigten

die Möglichkeit, sich zu Ihren Maßnahmen zu äußern. Dies

steigert die Mitarbeiterzufriedenheit, erhöht die Akzeptanz Ihrer

Maßnahmen und liefert Ihnen eine gute Datenbasis, um das

Erreichbarkeitsmanagement in Ihrem Unternehmen noch

effektiver zu gestalten. 


\subsubsection{Beispielhafte Maßnahmen für Beschäftigte}

Auch wenn der Arbeitgeber viel unternehmen kann, um ein sinnvolles Erreichbarkeitsmanagement in seinem Unternehmen zu fördern, funktionieren all diese Maßnahmen doch nicht ohne die Mitwirkung der Beschäftigten. Eine Zusammenstellung an möglichen Maßnahmen ist in Tab. 5.2 zu finden. Tab. 5.3 stellt zusätzliche Maßnahmen für Beschäftigte dar, die eine Führungsposition innehaben. Zu beiden Tabellen wird jeweils ein Beispiel für ein mögliches Tool gegeben.

\section{Beispiel für ein Tool: Reflexion der eigenen Erreichbarkeitspräferenzen}

Anna ist Sachbearbeiterin mit neun Jahren Berufserfahrung bei einem großen Finanzdienstleister. Sie arbeitet Teilzeit und pendelt vier Tage in der Woche 40 Minuten zu ihrer Arbeitsstelle. Der Job und die berufliche Verwirklichung ist ihr sehr wichtig, aber ihr Lebensschwerpunkt liegt außerhalb ihrer Arbeit. Sie ist Ehefrau, die sich den Haushalt mit ihrem Mann teilt, und darüber hinaus Mutter von zwei Töchtern im Teenageralter. Anna spielt zweimal pro Woche Volleyball in ihren Heimatverein, singt in einem Kirchenchor und engagiert sich als freiwillige Deutschlehrerin bei einer Abendschule für Flüchtlingskinder, wo sie einmal pro Woche Deutschunterricht gibt. Außerdem nimmt sich Anna jeden Tag etwas Zeit für sich, um zu meditieren, zu lesen oder sich einfach zu entspannen.

Schauen Sie sich die Lebenswelten von Anna sowie ihre Zeitaufteilung an. Wie würde das Bild für Sie aussehen? Welche Lebenswelten gibt es bei Ihnen, und wie verteilen Sie Ihre Zeit? Wie würden Sie Ihre Zeit gern verteilen? Skizzieren Sie Ihr eigenes Bild (Abb. 5.1).

\section{Beispiel für ein Tool: Wahrnehmung der Vorbildfunktion ${ }^{1}$}

Markus leitet ein Team aus acht Beschäftigten bei einem großen Automobilhersteller. Er ist Familienvater und arbeitet, wie sein Team, von Montag bis Freitag. Jeden Samstag fährt er seine Kinder um 9:30 Uhr zum Schwimmunterricht und nutzt die Zeit im Wartebereich, um sich auf die nächste Woche vorzubereiten: So checkt er beispielsweise seine Emails und gibt Feedback zu Fragen seines Teams. Nach einiger Zeit fällt ihm auf, dass seine Teammitglieder inzwischen fast direkt auf die Emails antworten, die er ihnen am Samstagvormittag sendet.

Was ist in diesem Beispiel passiert? Wie sollte Markus reagieren und wie würden Sie mit einer solcher Situation umgehen?

\footnotetext{
${ }^{1}$ An dieser Stelle ist erneut darauf hinzuweisen, dass das Arbeitszeitgesetz Vorgaben zur werktäglichen Arbeitszeit sowie zur Ruhezeit macht. Die Vorgaben zur Arbeitszeit können unter bestimmten Voraussetzungen verlängert sowie die Vorgaben zur Ruhezeit verkürzt werden. Details dazu finden sich in Kap. 2 sowie in Kap. 4.
} 
Tab. 5.2 Maßnahmen für Beschäftigte

\begin{tabular}{l|l}
\hline Titel der Maßnahme & Kurzerklärung der Maßnahme \\
\hline Reflexion der eigenen & Machen Sie sich klar, wie Sie erreichbar sein möchten. Dies ist \\
sehr wichtig und nicht so einfach, wie es vielleicht klingt. Wenn \\
Sie einen Wunsch frei hätten: Wann und wie würden Sie sich \\
wünschen, in Ihrer Freizeit für Ihre Arbeit erreichbar zu sein? \\
Und wie ist es für andere Lebensbereiche? Möchten Sie gern \\
nur für bestimmte Personen erreichbar sein, oder zum Beispiel \\
nur per Email?
\end{tabular}

\subsection{Erreichbarkeitsassistent für Smartphones}

Technischer Ansatz zur Umsetzung eines betrieblichen Erreichbarkeitsmanagements ist eine Software für Smartphones, der Erreichbarkeitsassistent. Zur Demonstration der technischen Umsetzbarkeit sowie der Erprobung und Evaluation der technischen Maßnahme wurde im Rahmen des Projekts ein Demonstrator entwickelt. Grundidee hinter dem Erreichbarkeitsassistenten ist es, eingehende E-Mails und Telefonate vor dem Zustandekommen bzw. der Zustellung zu prüfen und ggf. möglichst intelligent $\mathrm{zu}$ unterbinden bzw. zu verzögern (z. B. Zustellung der E-Mail von samstags nachts erst montags zu Beginn der Arbeitszeit). Der Erreichbarkeitsassistent besteht aus zwei Softwarekomponenten: einerseits einer App, welche auf dem Smartphone Nutzerinteraktion erlaubt und ggf. die Unterdrückung von Anrufen durchführt und andererseits einer Serverkomponente, die eingehende Kommunikation mit einem vorab definierten Regelwerk zum Erreichbarkeitsmanagement vergleicht und 
Tab. 5.3 Spezielle Maßnahmen für Führungskräfte

\begin{tabular}{l|l}
\hline Titel der Maßnahme & Kurzerklärung der Maßnahme \\
\hline Körderung der & Machen Sie Erreichbarkeitsmanagement zu einem Thema in Ihrem \\
Team. Sprechen Sie über Erwartungen und Wünsche. Erarbeiten Sie \\
gemeinsam eine gute Lösung und verbindliche Regeln. Halten Sie \\
die Regeln am besten schriftlich fest und diskutieren Sie sie \\
regelmäßig im Team, um einen Veränderungsbedarf aufzudecken.
\end{tabular}

E-Mails vor der Zustellung ggf. verzögert. Die Definition der Regeln kann individuell entsprechend der Anforderungen im Unternehmen mit einem Regeleditor vorgenommen werden, etwa im Rahmen einer Verhandlung zwischen Arbeitgeber und Betriebsrat. So können z. B. dienstliche E-Mails oder Telefonate in der Nacht entsprechend des Regelwerks unterdrückt bzw. zu einem späteren Zeitpunkt zugestellt werden. Für die Entscheidung, ob Kommunikation zugelassen, unterbunden oder verzögert wird, können je nach Verfügbarkeit im jeweiligen Unternehmen diverse Kriterien herangezogen werden, z. B. Beginn/Ende der Arbeitszeit, Rolle/Funktion (Mitarbeitende, Vorgesetzte, Geschäftsführung, Externe usw.) des Kommunikationspartners, belegte Zeiträume z. B. in einem Outlook-Kalender, direktes Nutzerfeedback und Einstellungen des Nutzers in der App.

\subsubsection{Umsetzung des Demonstrators}

Bei eingehender Kommunikation erfolgt im ersten Schritt die serverseitige Analyse, um die Funktionalität unabhängig von der verwendeten Client-Technologie (z. B. verwendeter E-Mail-Client) bereitstellen zu können. Dadurch bleibt der Empfangszeitpunkt einer 


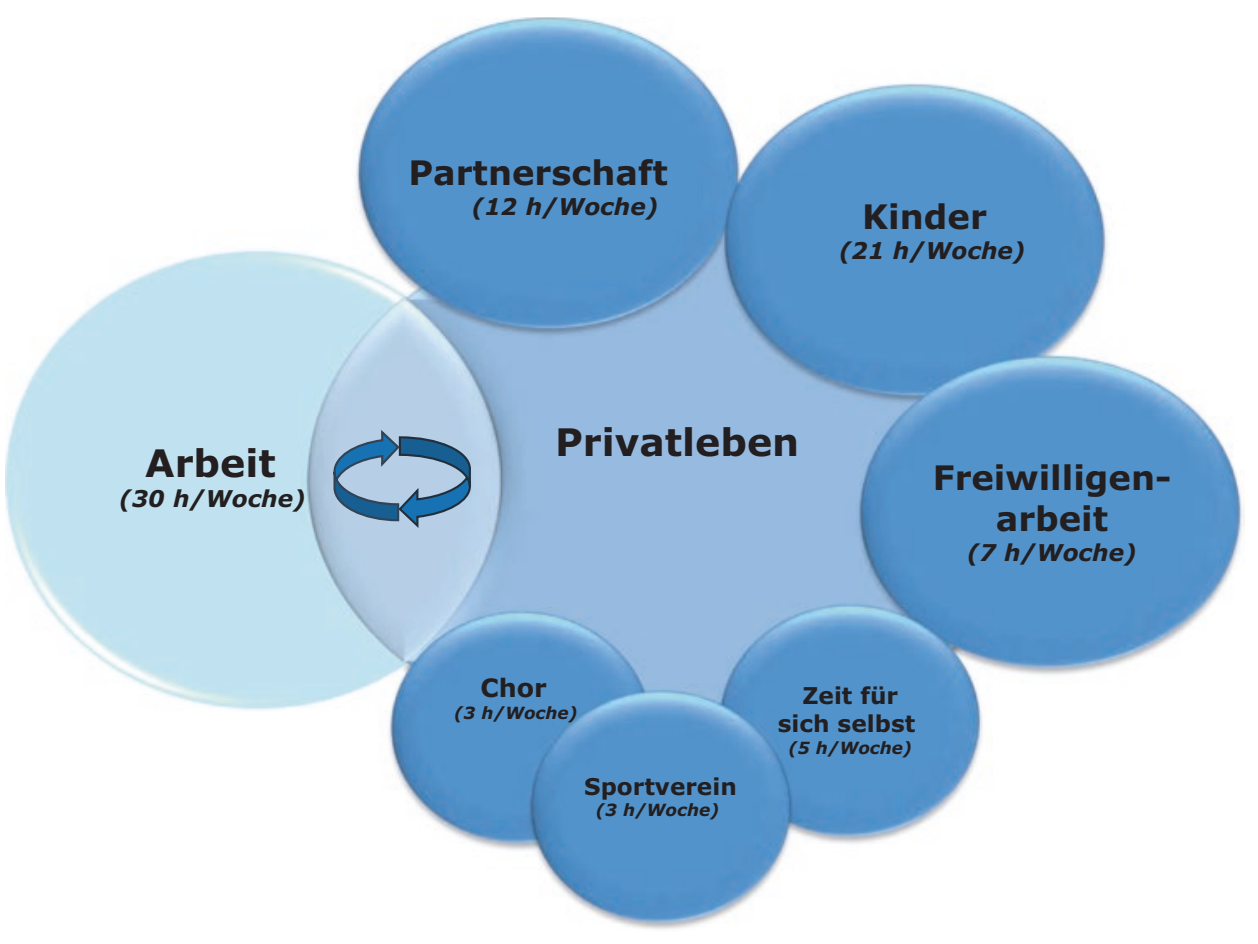

Abb. 5.1 Lebenswelten von Anna

E-Mail deterministisch und ist auf allen Abrufgeräten des Benutzers gleich, ohne dass eine spezielle Anwendung für jedes Gerät entwickelt werden muss. Zusätzlich bleibt der Einstellungsaufwand für den Mitarbeiter minimal, da die Serverkomponente die Funktion des bisherigen E-Mail Servers übernehmen und daher transparent für den Arbeitnehmer installiert werden kann. Die Serverkomponente des Demonstrators wird lokal bei dem Arbeitgeber installiert und ist für die Steuerung der Erreichbarkeit der Arbeitnehmer zuständig. Außerdem bietet sie einen zentralen Speicherort für die Benutzer Einstellungen und Kommunikationsregeln. Die zentrale Positionierung der Serverkomponente ermöglicht es, E-Mail-Kommunikation bereits vor Erreichen des Postfachs des Empfängers abzufangen und entsprechend der Benutzereinstellungen zu verzögern (Abb. 5.2).

Die Informationsbereitstellung der für die Entscheidungsfindung erforderlichen Daten erfolgt über einzelne Dienste, welche Daten jeweils aus bestimmten Quellen beschaffen, z. B. durch Auslesen von Urlaubszeiträumen aus den digitalen Kalendern von Mitarbeitern. Durch die Unterteilung in einzelne Dienste wird sowohl die Erweiterbarkeit durch Einbindung weiterer Dienste ermöglicht als auch das komplette Entfernen von Funktionalitäten, die im jeweiligen Anwendungsumfeld nicht erwünscht oder nicht erforderlich sind, wie z. B. die Ortung von Mitarbeitern über ihr Smartphone. Auf Basis der so verfügbar gemachten Daten kann durch Definition von Regeln erfolgen. 


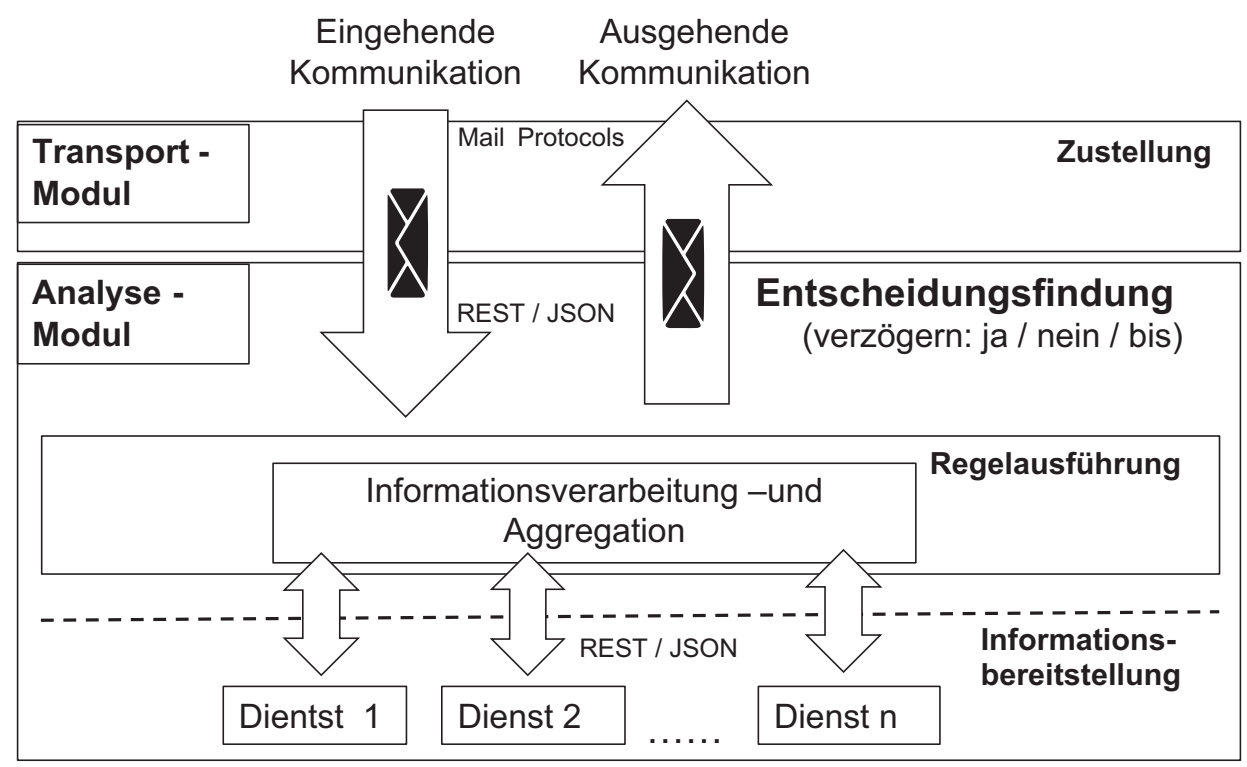

Abb. 5.2 Architekturüberblick Erreichbarkeitsassistent

Ausgehend vom definierten Regelwerk erfolgt dann nach der Auswertung der Regeln die automatische Entscheidungsfindung. Darauf basierend erfolgt entsprechend die Zustellung bzw. die Verzögerung der Zustellung. Das System bietet zudem die Möglichkeit, eingehende E-Mails nicht direkt an den designierten Empfänger weiterzuleiten und stattdessen nutzerdefiniert bis zu einem vorherbestimmten Zeitpunkt zurückzuhalten. Dies ermöglicht das Definieren von Nichterreichbarkeitszeiträumen, in welchen keine Zustellung von eingehender Kommunikation erfolgt. Stattdessen werden eingehende E-Mails bis Ende dieses Zeitraums zurückgehalten und schließlich gesammelt zugestellt.

Die gebündelte Zustellung funktioniert analog zu den Nichterreichbarkeitszonen, verfolgt aber das Ziel dem Nutzer Phasen der Konzentration zu ermöglichen, in denen dieser nicht durch eingehende E-Mail-Kommunikation abgelenkt wird. Dabei kann der Nutzer das System so konfigurieren, dass E-Mails lediglich periodisch (z. B. stündlich) zugestellt werden. Dies bedeutet, E-Mails, die während dieser Periode eingehen werden, zuerst gesammelt und am Ende der aktiven Periode zugestellt.

\subsubsection{Weiterführende Funktionalitäten}

Für die automatische Klassifikation eingehender E-Mails werden Techniken des maschinellen Lernens erprobt. Diese Systeme verwenden verschiedene Attribute der eingehenden Nachrichten (z. B. Absender und Empfänger, Betreff, Inhalt oder Absendezeitpunkt) und versuchen daraus weitere Attribute der Nachricht automatisch zu bestimmen. Diese zu bestimmenden Attribute können beispielsweise die Zugehörigkeit zu einem bestimmten Projekt oder die Dringlichkeit (auf einer diskreten oder kontinuierlichen Skala) der 
Nachricht sein. Diese automatisch bestimmten Attribute können dann in Regeln verwendet werden, um die Entscheidung des Systems zu beeinflussen.

Dies erlaubt dem Nutzer beispielsweise das Erstellen von Regeln, welche an bestimmten Tagen die Kommunikation für ein bestimmtes Projekt sofort durchstellt, während andere Nachrichten nur gesammelt zugestellt werden. Ein weiterer Anwendungsfall ist das Umgehen von Nichterreichbarkeitszeiten für als sehr dringend eingestufte E-Mails.

Die Ansätze, die im Demonstrator getestet werden, basieren auf Maschinellem Lernen sowohl zur Vorverarbeitung der Nachrichten und zur Entscheidungsfindung. Klassischerweise benötigen diese Deep-Learning Ansätze eine große Menge an Trainingsdaten, um ein gut generalisierendes Modell zu lernen, welches auch in der Lage ist bisher ungesehene Datensätze korrekt einzuordnen. Diese Trainingsdaten bestehen aus der Eingabe in das System, so wie der Sollentscheidung, welche das System für dieses Datum ausgeben soll. Gerade aber diese Sollausgabe ist oft nicht in großen Mengen für das konkrete Problem vorhanden. Daher werden im Rahmen des Demonstrators mehrere Ansätze entwickelt und evaluiert, welche das Problem der geringen Trainingsmenge relativieren. Diese Ansätze umfassen das vorverarbeiten der Eingabedaten, z. B. durch Training eines generellen Sprachmodells das die Eingabe normalisiert und dadurch den Raum der möglichen Eingaben, welcher im Falle natürlicher und daher unstrukturierter Sprache sehr groß ist, versucht zu verkleinern (Mikolov et al. 2013; Devlin et al. 2018). Ein weiterer Ansatz ist das Transferlernen aus Daten, die aus einer ähnlichen Domäne stammen, aber die gesamt vorhandene Datenmenge vergrößert (Grießhaber et al. 2018). Normalerweise können Daten aus unterschiedlichen Domänen nicht einfach für das Training eines Modells verwendet werden, da dadurch das Modell sich eventuell zu stark an die Daten der „falschen“ Domäne anpasst (overfitting) (Ganin et al. 2015).

Für den Fall, dass viele Trainingsdaten vorhanden sind, diese aber nicht über die Sollausgabe verfügen, gibt es außerdem das Konzept des ,,aktiven Lernens“ bei dem das Modell mit einem kleinen Set an Trainingsdaten anfängt und dabei herausfindet welche Trainingsdaten durch den Nutzer mit einer Sollausgabe annotiert werden sollen um das Training am schnellsten voranzubringen. Dadurch wird der Aufwand des Nutzers möglichst geringgehalten, während sich das Modell möglichst effizient über die Zeit verbessern kann (Olsson 2009).

\subsubsection{Einholung von Nutzerfeedback}

Die Serverkomponente kann jeder eingehenden E-Mail eine Fußzeile hinzufügen, die es dem Nutzer erlaubt, dem System Feedback über dessen Entscheidung zu geben. Dieses Feedback erfolgt in Form einer einfachen, binären Entscheidung: Daumen hoch (positiv) oder runter (negativ). Das Feedback kann zur Verbesserung des Systems an mehreren Stellen genutzt werden. Einerseits kann die globale Auswertung des Feedbacks aller Nutzer dafür verwendet werden, um die Effektivität des Gesamtsystems zu evaluieren und die Konfiguration des Systems zu verbessern. Andererseits kann die Entscheidung für ein neues Trainingsdatum für das Training der automatischen Klassifikationssysteme des datenbasierten Ansatzes verwendet werden (Abb. 5.3). 


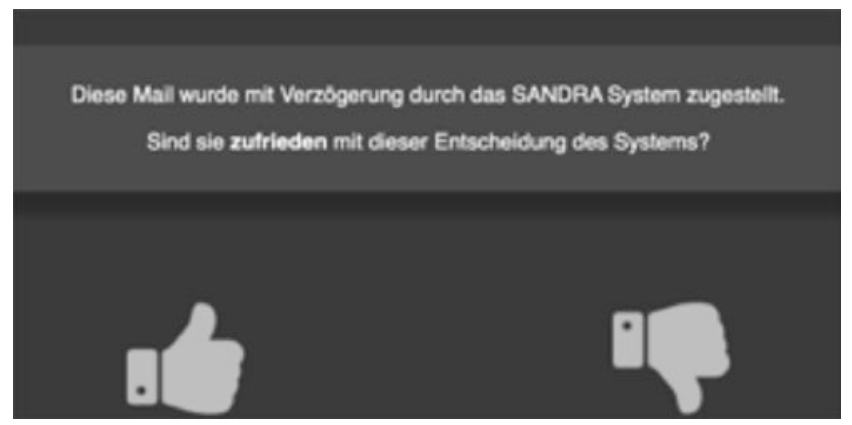

Abb. 5.3 Footer unter einer, durch das System verzögert zugestellten E-Mail

\subsubsection{App}

Jeder Benutzer des Systems hat die Möglichkeit sich verschiedene Profile anzulegen. Zu jedem Zeitpunkt ist eines dieser Profile aktiv. Abhängig vom ausgewählten Profil können verschiedene Erreichbarkeitseinstellungen mit unterschiedlichen Konfigurationen aktiv sein. Profile können dabei automatisch in Abhängigkeit verschiedener Bedingungen aktiviert, oder manuell durch den Nutzer ausgewählt werden. Die automatische Umschaltung kann beispielsweise durch die aktuelle Tageszeit oder durch Kalendereinträge erfolgen. Die manuelle Auswahl eines Profils kann jeder Zeit durch die Smartphone App erfolgen.

Die Smartphone App bietet dem Nutzer die Möglichkeit zur Personalisierung des Erreichbarkeitsmanagers durch Konfiguration des Verhaltens der Serverkomponente. Außerdem ist die App technisch für die Umsetzung der Anrufblockierung notwendig.

Für den Demonstrator wurde die Funktionalität lediglich für das Android Betriebssystem prototypisch entwickelt.

\subsubsection{Visualisierung und Anpassung der aktuellen Einstellungen}

Eine der Aufgaben der App ist die Visualisierung der aktuellen Erreichbarkeitseinstellungen. Dies umfasst das aktuell aktivierte Profil, sowie damit zusammenhängende Detaileinstellungen wie aktuell blockierte Telefonnummern, aktive und automatisierte E-MailVerzögerungen oder Kalendereinträge die u. U. die Entscheidung des Systems beeinflussen.

Diese Visualisierung dient nicht nur der Information des Nutzers. Vielmehr ist die Nachvollziehbarkeit der Entscheidungen, welche das System trifft, ein rechtliches Grundkriterium nach Erwägungsgrund 58 der DSGVO.

Neben dem Webinterface der Serverkomponente sollen die Erreichbarkeitseinstellungen des Systems auch über die mobile App anpassbar sein. Dies ermöglicht es dem Nutzer ein optimiertes Userinterface bereitzustellen, welches Bedienkonzepte verwendet die dieser bereits aus anderen mobilen Anwendungen gewohnt ist.

Außerdem können über die App zusätzliche Informationen zu den Einstellungen bereitgestellt werden. Wird beispielsweise eine Einstellung geändert, welche nach dem Grundsatz der DSGVO zusätzliche Daten des Nutzers benötigt, wird dieser über ein Popupdialog da- 
rüber informiert und zum expliziten Opt-in aufgefordert. Außerdem kann der Nutzer zusätzliche Informationen zur Funktionalität und Bedeutung bestimmter Einstellungen aufrufen.

\subsubsection{Blockieren eingehender Anrufe}

Versionen des Android Betriebssystems vor „Nougat“ (Versionsnummer 7, Veröffentlichung 2016) ${ }^{2}$ boten keine vereinheitlichte Schnittstelle zur Blockierung eingehender Anrufe (Google Inc. 2019). Da diese Schnittstellen jedoch für die Umsetzung des telefonischen Erreichbarkeitsmanagements im Demonstrator vorhanden sein müssen wurde diese Version als Mindestvoraussetzung zur Implementierung der App verwendet. Stand Mai 2019 ist diese oder eine neuere Version bereits auf über der Hälfte aller Smartphones mit dem Android Betriebssystem installiert. ${ }^{3}$

Des Weiteren bieten diese Schnittstellen lediglich die Möglichkeit eingehende Anrufe auf Basis einer Filterliste automatisch abzuweisen (Blacklisting). Es ist daher nicht möglich, eingehende Anrufe generell zu blockieren, umzuleiten oder den Anrufer darauf hinzuweisen, wann der Kontakt wieder verfügbar ist.

Im Demonstrator ist daher das explizite Blockieren von eingehenden Anrufen von bestimmten Nummern möglich. Jede Filterliste ist dabei einem Profil zugeordnet, was das automatische Umschalten der aktivierten Filterliste auf Basis der aktuellen Tageszeit oder eines aktiven Kalendereintrags ermöglicht. Dadurch kann z. B. der Anwendungsfall umgesetzt werden, außerhalb der Arbeitszeiten nicht für geschäftliche Kontakte erreichbar zu sein. Da die Telefonnummern explizit in der Filterliste hinterlegt werden müssen, ist es außerdem trivial Ausnahmen zu solchen Regeln hinzuzufügen und beispielsweise Anrufe von Familienmitgliedern nie zu blockieren.

\subsubsection{Auslesen von Kalendereinträgen}

Über die App hat der Anwender die Möglichkeit, das aktuelle Profil automatisch zu wechseln, während ein Kalendereintrag mit einem definierbaren Betreff stattfindet. Dies ermöglicht dem Anwender beispielsweise die automatische Erreichbarkeitsverwaltung basierend auf dem Urlaubsstatus, abhängig von aktuell stattfindenden Meetings oder Gleitfreizeiten.

Die App bietet dafür nicht nur die Möglichkeit zur Konfiguration der Funktionalität, sondern hat auch die Aufgabe auf die Kalender des Smartphones zuzugreifen, um etwaige Kalendereinträge zu finden. Dieser Ansatz vermeidet das Verwalten eines speziellen „Erreichbarkeitskalenders“ auf der Serverseite, stattdessen kann der Nutzer wie gewohnt seinen bisherigen Kalender nutzen.

\subsubsection{User Interface}

Bei der Gestaltung der App wurden gängige Design Prinzipien und Normen, wie die ISO 9241-110 berücksichtigt. Insbesondere um der Erwartungskonformität der Nutzer gerecht

\footnotetext{
${ }^{2}$ https://developer.android.com/about/versions/nougat/index.html.

${ }^{3} \mathrm{https}: / /$ developer.android.com/about/dashboards.
} 
zu werden, wurden gängige UI Komponenten eingesetzt. Diese sind den Nutzern durch Verwendung anderer Apps bereits vertraut und müssen so nicht neu gelernt werden.

Öffnet der Nutzer die App bekommt er das aktuell aktive Profil angezeigt (Abb. 5.4).

Im Header werden die wichtigsten Infos des Profils kurz dargestellt (dies sind auch die Informationen, die der Nutzer als Notification auf seinem Smartphone angezeigt bekommt).

Unterhalb des Headers werden alle Eigenschaften und Einstellungen des Profils angezeigt.

Der Nutzer hat die Möglichkeit das Profil manuell zu wechseln.

Hierzu tippt er auf den Kreis-Button unten rechts und bekommt dadurch alle verfügbaren Profile angezeigt (Abb. 5.5).

Über das Burger-Menü oben rechts, gelangt der Nutzer zur Profilübersicht, Kontoverwaltung, den Einstellungen und seinem Benutzerkonto. Diese Menü-Punkte stellen lediglich einen Vorschlag dar und können je nach Bedarf noch angepasst werden.

Unter dem Menüpunkt „Profile“, erhält der Nutzer die Möglichkeit seine Profile zu verwalten, sie zu bearbeiten oder ein neues Profil hinzuzufügen.

\subsubsection{Testbetrieb und Evaluation}

Wie im vorangegangenen Abschnitt beschrieben, dient der Demonstrator zur Sicherstellung der Umsetzbarkeit der im Projekt erarbeiteten technischen Maßnahmen, sowie der

Abb. 5.4 Aktuell aktives Profil in der App

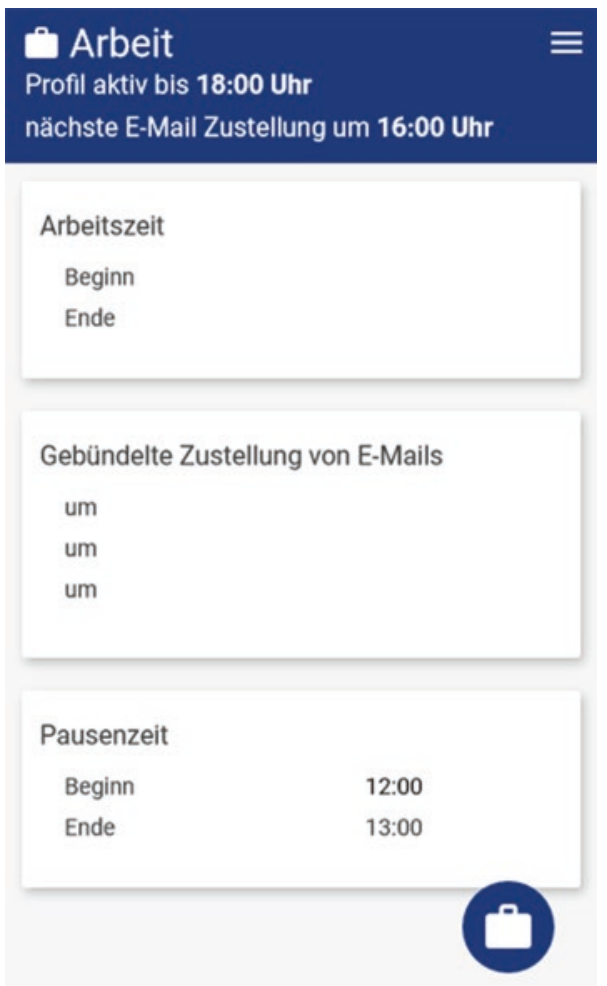


Abb. 5.5 Manuelle Auswahl eines anderen Profils in der App

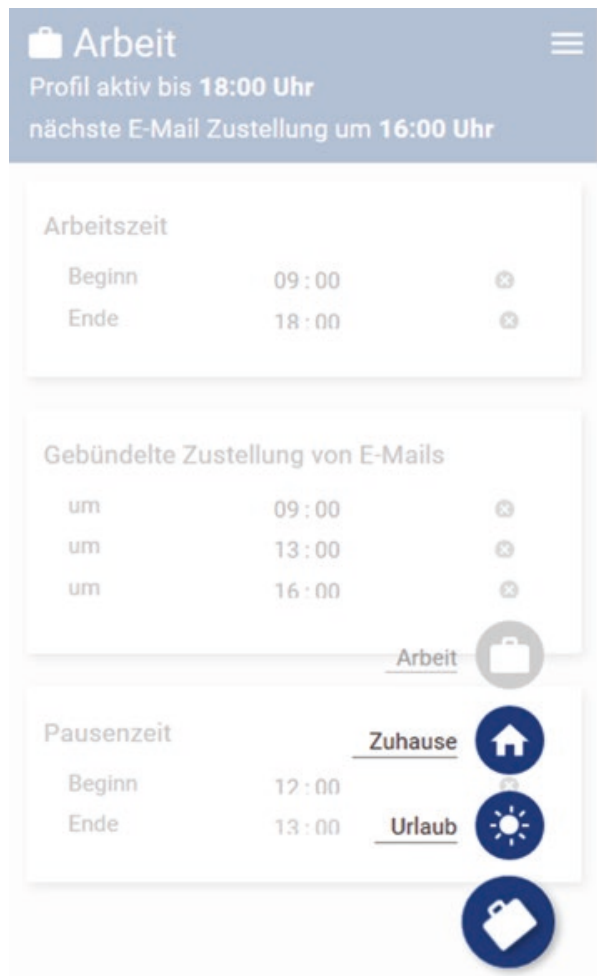

Evaluation der Effektivität dieser Maßnahmen in der Praxis. Dazu wurden bereits früh in der Entwicklung zwei Anwendungsunternehmen aus verschiedenen Branchen für einen Pilotbetrieb des Systems einbezogen.

Die AGILeVIA GmbH ist ein technologieorientiertes Beratungsunternehmen mit Wurzeln in der angewandten Forschung mit dem Schwerpunkt auf Prozesse, beginnend bei der Ideenfindung, über die Erprobung und Serieneinführung, bis hin zur Marktdurchdringung für Produkte und Dienstleistungen.

Die AK Reprotechnik GmbH ist ein Unternehmen aus der Medienbranche mit Fokus auf Print on demand, die vorzugsweise die Automobilindustrie mit fahrzeugbegleiteter Literatur Just-in-Time beliefert.

\subsubsection{Messverfahren}

Die Evaluation der implementierten Erreichbarkeitsmaßnahmen wird in zwei getrennten Dimensionen gemessen. Die funktionale Messung verwendet Nutzer Feedback, um eine quantitative Auswertung der subjektiven Zufriedenheit mit den umgesetzten technischen Erreichbarkeitsmaßnahmen zu ermöglichen.

Um neben diesen subjektiven Einschätzungen der Nutzer auch objektive Ergebnisse zu erhalten, wurden für die Evaluation Methoden zur Stressmessung entwickelt, mit der Ab- 
sicht dadurch einen Einblick in das Stresslevel der Testanwender zu erhalten. Diese Dimension der Auswertung begründet sich aus dem Ziel der allgemeinen Stressminderung durch das Erreichbarkeitsmanagement des Projektes.

Ein weiteres Ergebnis der Evaluation ist die Validierung der konzipierten Systemarchitektur in Bezug auf Umsetzbarkeit und Anwenderfreundlichkeit.

\subsection{Funktionale Messung}

Die funktionale Messung basiert auf Rückmeldung der Nutzer. Dafür wurde die Funktionalität des Demonstrators genutzt, bei jeder eingegangenen und zugestellten E-Mail über eine Fußzeile dem System Feedback zu geben ob der durch das System gewählte Zustellzeitpunkt korrekt war. Da E-Mails entweder sofort oder verzögert zugestellt werden, ergibt sich der Werteraum wie in Tab. 5.4 dargestellt.

Durch diese Zuordnung können für die quantitative Analyse die statistischen Kennzahlen aus Tab. 5.5 errechnet werden.

Durch die Zuordnung von positivem Feedback zu den positiv-Kategorien beschreibt die Genauigkeit bei dieser Auswertung die subjektive Zufriedenheit des Nutzers mit der Entscheidung des Erreichbarkeitsmanagers.

Die Kennzahl der Präzision beschreibt in diesem Kontext wie häufig das System richtigerweise die Entscheidung getroffen hat, eine eingehende Nachricht erst verzögert zuzustellen. Sie wird daher auch als positiver Vorhersagewert beschrieben.

Die Sensitivität ist ein Maß dafür, wie wahrscheinlich es ist, dass das System eine Nachricht, welche der Nutzer mit Verzögerung zugestellt haben will, auch wirklich verzögert.

Das F1-Maß ist ein Kombinationsmaß aus der Präzision und der Trefferquote. Da beide Kennzahlen die Güte der Entscheidungen beschreiben, wird durch die Kombination als harmonisches Mittel eine weitere relevante Kennzahl gebildet, die beide Aspekte abbildet.

Generell liefern alle beschriebenen Maße eine Zahl im Wertebereich von 0 bis 1, wobei Zahlen nahe 1 ein positives Ergebnis der Auswertung darstellen. Da während der Entwicklung des Demonstrators mehrere Messungen durchgeführt werden, deutet eine positive Entwicklung aller Kennzahlen auf die Einführung effektiver und funktionaler technischer Lösungen hin.

Tab. 5.4 Möglicher Wertebereich des Userfeedbacks

\begin{tabular}{l|l|l}
\hline & verzögerte Zustellung & sofortige Zustellung \\
\hline positives Feedback & wahrpositiv (WP) & wahrnegativ (WN) \\
\hline negatives Feedback & falschpositiv (FP) & falschnegativ (FN) \\
\hline
\end{tabular}

Tab. 5.5 Auflistung der statistischen Kennzahlen zur quantitativen Auswertung des Userfeedbacks

\begin{tabular}{l|l|l|l}
\hline Genauigkeit & $\begin{array}{l}\text { Präzision (Olson } \\
\text { and Delen 2008) }\end{array}$ & $\begin{array}{l}\text { Trefferquote } \\
\text { (Yerushalmy 1947) }\end{array}$ & $\begin{array}{l}\text { F1-Maß (Yerushalmy } \\
\text { 1947) }\end{array}$ \\
\hline$A C C=\frac{W P+W N}{W P+W N+F P+F N}$ & $P=\frac{W P}{W P+F P}$ & $S=\frac{W P}{W P+F N}$ & $F=\frac{2 * W P}{2 * W P+F P+F N}$ \\
\hline
\end{tabular}




\subsection{HRV Analyse zur Stressmessung}

Akuter und chronischer Stress, insbesondere auch der durch Arbeit hervorgerufene chronische Stress, wirken sich vor allem auf das Autonome Nervensystem (ANS) aus (Henry 1997; Schroeder et al. 2003; Togo and Takahashi 2009). Die in Togo and Takahashi (2009) analysierten Studien stimmen darin überein, dass Arbeitsstress zu Veränderungen der Regulationsmechanismen des ANS führen, die sich nicht nur während der Arbeitszeit, sondern auch während des Nachtschlafs nachweisen lassen. Messbar sind die stressbedingten Veränderungen des ANS über die Herzratenvariabilität (HRV). Die American Heart Rate Association hat in Schroeder et al. (2003) Empfehlungen für die zuverlässige Messung der HRV-Parameter definiert. Diese Empfehlung gilt als quasi-Standard für die nicht-invasive HRV-basierte Stressindikation. U. a. ist darin auch die HRV-Bestimmung aus während des Nachtschlafs aufgenommenen Langzeitmessungen definiert.

Generell müssen für die Bestimmung der HRV-Parameter die RR-Abstände zwischen den Herzschlägen gemessen werden. Für diese Messung braucht es keine teuren EKGGeräte. Wie z. B. in Togo and Takahashi (2009) nachgewiesen wurde, können die RRIntervalle mit handelsüblichen Brustgurten, z. B. der Firma Polar, EKG-genau gemessen werden.

Das Autonome Nervensystem (ANS) ist der Teil des Nervensystems, der unserer willentlichen Kontrolle weitestgehend entzogen ist. Über das Autonome Nervensystem werden lebenswichtige Funktionen gesteuert. Hierzu gehört z. B. die Kontrolle der Atmung, der Verdauung, des Stoffwechsels und des Herz-Kreislaufsystems.

Unterteilt ist das ANS in zwei Bereiche:

- Das sympathische System sorgt dafür, dass wir in entsprechenden Situationen maximal leistungsfähig werden. Das sympathische System lässt das Herz schneller schlagen und sorgt für erhöhte Blutzufuhr zu Muskeln, Herz und Gehirn. Es erhöht den Blutzuckerspiegel und hemmt gleichzeitig andere Funktionen wie Verdauung, Wachstum, Nierentätigkeit oder Insulinsekretion.

- Das parasympathische System sorgt für Erholung, Entspannung und Wiederherstellung der Kräfte nach starker Belastung. Dafür verringert es z. B. die Herzrate, regt zur Nahrungsaufnahme und Verdauung an und verbessert die Immunreaktivität.

Bei einem gesunden Menschen sollte ein harmonisches Gleichgewicht zwischen parasympathischer und sympathischer Aktivierung herrschen. D. h. unter anderem, dass unser Körper sehr wohl in der Lage ist außergewöhnliche Leistung zu erbringen, aber nur wenn wir rechtzeitig dem parasympathischen System die Chance geben, Erholung und Regeneration anzustoßen. Eine Vielzahl physischer und psychischer Krankheiten wird dadurch verursacht, dass das parasympathische System nicht mehr rechtzeitig und ausreichend zum Einsatz kommt.

Die Analyse der HRV ermöglicht die Messung der parasympathischen und sympathischen Aktivierung und damit eine Früherkennung pathogener Zustände. 
Für die Evaluation der Stressminderung wurde im Rahmen des Projekts eine Smartphone App zur Messung der HRV-Parameter implementiert. Die App empfängt die vom Brustgurt aufgenommenen RR-Intervalle über Bluetooth. Die Messung wird von der Smartphone-App an einen Server übertragen. Dort werden die HRV-Parameter berechnet. Ihr zeitlicher Verlauf kann über den Browser analysiert werden (Abb. 5.6).

Im Forschungsprojekt SANDRA wird die HRV-Messung eingesetzt, um die Auswirkung des Erreichbarkeitsmanagements auf die Erholung während des nächtlichen Schlafs zu messen. Die Hypothese ist, dass sich durch den Verzicht auf berufliche Kommunikation nach Feierabend, die HRV früher dem Erholungspegel annähert als im gegenteiligen Fall. Dazu werden Mitarbeiter der an der Evaluation teilnehmenden Partnerunternehmen unter beiden Prämissen - mit und ohne berufliche Korrespondenz am Abend - HRV-Messungen über die Nacht durchführen. Dafür sollte die Messung beim Zubettgehen gestartet und am nächsten Morgen unmittelbar nach Erwachen beendet werden. Nach dem Hochladen der Messung auf den Server und der Berechnung der HRV Parameter, kann diese über den Webbrowser analysiert werden.

Die HRV-Parameter nehmen nicht nur durch Belastung bzw. Stress am Vorabend niedrige Werte an, sondern auch durch

- Bewegung während der Messung

- Husten und Nießen während der Messung (temporär)

- Alkoholkonsum vor der Messung

- Üppiges und spätes Essen vor der Messung

- Körperliche Belastung (Sport) in den Stunden vor der Messung

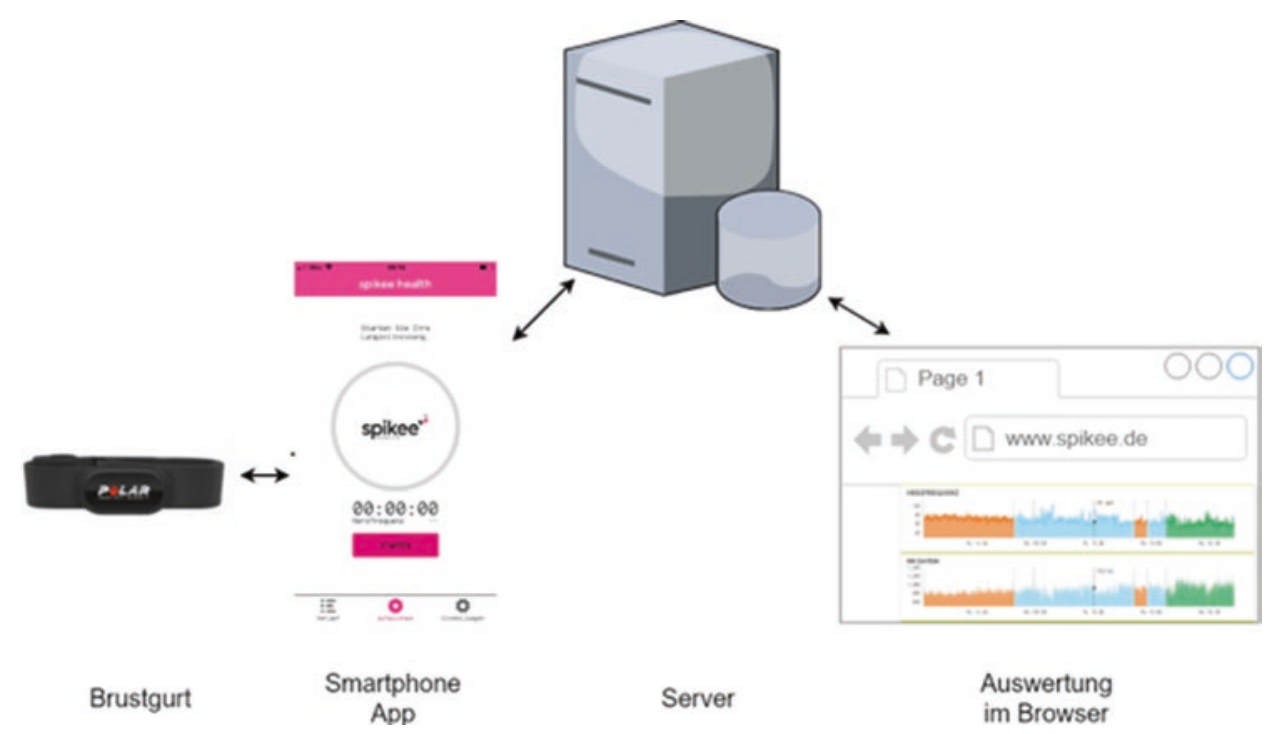

Abb. 5.6 Systematische Architektur des im Projekt eingesetzten System zur Stressmessung 
- Erkältungen, grippale Effekte und andere Krankheiten

- Medikamenteneinnahme

Für die Studie im Rahmen des SANDRA Projektes ergibt sich daraus die Rahmenbedingung, an den Messtagen die oben genannten Faktoren auszuschließen bzw. konstant zu halten, sodass die variierende geschäftliche Email-Kommunikation am Abend als einzige Einflussgröße angenommen werden kann.

\subsubsection{Einführung und Optimierung}

$\mathrm{Da}$ es bei der Erprobung komplexer Systeme immer zu unerwarteten Problemen im Betrieb kommen kann, wurde bei der Einführung darauf geachtet, dass der normale Betrieb in den Partnerunternehmen sichergestellt ist. Dies ist besonders wichtig, da sich eine Störung der Kommunikation potenziell geschäftsschädigend auswirken kann. Daher wird, besonders in der Anfangszeit, der Demonstrator parallel zu den bisherigen E-Mailsystemen betrieben. In dieser Phase können anfängliche Fehler ohne negative Folgen für die Partnerunternehmen, z. B. durch verloren gegangene E-Mails, identifiziert und behoben werden. Mit voranschreitender Reife des Systems kann der Übergang auf den Demonstrator fließend erfolgen, bis schließlich die alten E-Mailserver gänzlich, durch das Erreichbarkeitsmanagement System gesteuerten Dienste ersetzt werden können.

Beide Partnerunternehmen verwenden bereits externe IT-Dienstleister für die Verwaltung ihrer Onlinekommunikation, sodass die Unternehmen selbst keine IT-Infrastruktur dafür vor Ort besitzen. Für die Bereitstellung der Serverkomponente wurden aus diesem Grund für jeden Projektpartner getrennte virtuelle Server bei einem externen Dienstleister gebucht (Hostingprovider). Bei der Auswahl des Anbieters wurde aus datenschutzrechtlichen Gründen besonders darauf Wert gelegt, dass sich nicht nur der Sitz des Unternehmens innerhalb Deutschlands befindet, sondern auch die Infrastruktur in innerländischen Rechenzentren bereitgestellt wird. Dadurch kann sichergestellt werden, dass schützenswerte Daten in Form von Kommunikationsprotokollen oder E-Mailnachrichten nie zur Verarbeitung in das Ausland transferiert werden müssen. Obwohl dies im Evaluationsbetrieb durchaus nach expliziter Zustimmung der Partnerunternehmen möglich wäre, ist dadurch sichergestellt, dass alle Daten beim Anbieter DSGVO-konform abgespeichert werden können. Neben diesen datenschutzrechtlichen Gründen hat ein Standort in Deutschland noch zusätzliche Vorteile hinsichtlich der Datensicherheit und Geschwindigkeit (Jäger 2018).

\subsubsection{Kontinuierliche Einführung neuer Funktionen}

Die Entwicklung des Demonstrators erfolgt nach dem Prinzip der agilen Softwareentwicklung, bei der jede neue Funktion oder Komponente schnellstmöglich in das Produktionssystem eingeführt wird (Continuous Deployment [CD]). Außerdem wird durch automatisierte Tests sichergestellt, dass neue Funktionen erwartungsgemäß funktionieren (Komponententests) und keinen negativen Einfluss auf die Arbeitsfähigkeit der bereits vorhandenen Komponenten haben (Regressionstest). Dieser Prozess wird auch als Continuous Integration (CI) bezeichnet. 
Die Verwendung der CI/CD Praktiken ermöglicht einen kurzen Feedbackzyklus der Partnerunternehmen bei Einführung neuer Funktionen mit geringstmöglicher Störung des laufenden Betriebs (Shahin et al. 2017).

\subsection{Evaluationsplan}

In der ersten Evaluationsphase des Pilotbetriebs wurde zuerst ein simples und starres Regelwerk im Erreichbarkeitsmanager implementiert, ohne die Anpassung der Parameter durch den Nutzer zu ermöglichen. Dieses Regelwerk wurde durch die Partnerunternehmen spezifiziert und bildet die üblichen Geschäftszeiten in diesen ab.

In diesem Regelwerk wird zwischen drei Erreichbarkeitsstufen unterschieden:

1. Volle und uneingeschränkte Erreichbarkeit während der gesamten Kernarbeitszeit

2. Eingeschränkte Erreichbarkeit für bestimmte Kontakte

3. Störungsfreie Nachtruhe

Bei voller Erreichbarkeit werden alle E-Mails sofort und ohne Verzögerung zugestellt. Während der Nachtruhe werden alle eingehenden E-Mails bis zum nächsten Morgen zurückgehalten und, abhängig von der Sendeadresse, entweder bei Beginn der eingeschränkten Erreichbarkeit, spätestens aber mit Beginn der Kernarbeitszeit zugestellt.

Die eingeschränkte Erreichbarkeit bildet im Falle des Partnerunternehmens AGILeVIA $\mathrm{GmbH}$ den Anwendungsfall ab, außerhalb der Kernarbeitszeiten nur für bestimmte Kontakte erreichbar zu sein. Im konkreten Fall sind das internationale Geschäftspartner für welche, durch ihren Sitz im Ausland und der daher auftretenden Zeitverschiebung durch diese Erreichbarkeitsregeln ein realistischeres Kommunikationsfenster geschaffen wird. Eingehende Kommunikation dieser Geschäftskontakte wird während der eingeschränkten Erreichbarkeit ohne Verzögerung zugestellt, E-Mails von Absendern außerhalb dieser Ausnahmen werden, wie zur Nachtruhenzeit, bis zum Beginn des nächsten Geschäftstages zurückgehalten. In der nächsten Evaluationsphase wurde es mit Einführung der Smarthone Applikation dem Nutzer ermöglicht, das in der vorherigen Phase eingeführte Regelwerk zu personalisieren. Dazu wurde die Möglichkeit geschaffen, die Anfangs- und Endzeiten der jeweiligen Erreichbarkeitsstufen zu wählen.

Nach der initialen Einführung der App und Bereitstellung von Testgeräten für die Evaluationspartner ist es möglich durch automatisierte Over-the-Air (OTA) Updates der App und die oben beschriebene Verwendung von CI/CD Techniken bei der Entwicklung der Serverkomponente, neue Funktionen des Demonstrators feingranularer und häufiger zu veröffentlichen. Durch die enge Zusammenarbeit zwischen den Evaluations- und Entwicklungspartnern ermöglicht dies eine iterative Verbesserung der Funktionalität. In den darauffolgenden Phasen wird der Erreichbarkeitsmanager im Folgenden erweitert. Eine Besonderheit dabei ist die Funktion der ,gebündelten Zustellung“ da diese im Partnerunternehmen AGILeVIA GmbH bereits als organisatorische Maßnahme in Form der 52/17 Regel (Bradberry 2015) umgesetzt ist. Diese Funktion dient daher als Beispiel für eine technische Maßnahme, die eine organisatorische Maßnahme ersetzt und somit im Idealfall den Mehraufwand der Selbstorganisation reduziert. 


\subsection{Fazit}

Im Forschungsprojekt SANDRA werden sowohl ein Erreichbarkeitsassistent als auch organisatorische Maßnahmen in zwei Anwender-Unternehmen aus zwei verschiedenen Branchen erprobt. Die bisherigen Erkenntnisse aus dem Testbetrieb zeigen, dass sich durch eine technische Lösung Maßnahmen zur Verringerung der Belastung durch ständige Erreichbarkeit über Smartphones technisch umsetzen lassen und dass von einer Verringerung der Belastung durch ständige Erreichbarkeit über Smartphones ausgegangen werden kann. In der noch ausstehenden Evaluation werden mit den im Kapitel beschriebenen Verfahren sowohl die technische Leistungsfähigkeit des Erreichbarkeitsassistenten als auch die Stressreduktion durch Implementierung sämtlicher Maßnahmen (organisatorisch und technisch) gemessen, um belastbare Aussagen über Nutzen und Grenzen eines betrieblichen Erreichbarkeitsmanagements treffen zu können.

Für die Zukunft stellt sich zudem die Frage, ob weitere Kommunikationstechnologien in ein Erreichbarkeitsmanagement einzubeziehen sind. Hierbei relevant erscheinen aufgrund ihrer gestiegenen Verbreitung im Umfeld dienstlicher Kommunikation, z. B. Instant Messanging Systeme oder auch Varianten aus den Social Media - Umfeld.

\section{Literatur}

Ayyagari, R., Grover, V., \& Purvis, R. L. (2011). Technostress: Technological antecedents and implications. MIS Quarterly, 35, 831. https://doi.org/10.2307/41409963.

Bradberry, T. (2015). Why the 8-hour workday doesn't work. https://www.linkedin.com/pulse/perfect-amount-time-work-each-day-dr-travis-bradberry. Zugegriffen am 15.09.2019.

David, K., Bieling, G., Bohnstedt, D., et al. (2014). Balancing the online life: Mobile usage scenarios and strategies for a new communication paradigm. IEEE Vehicular Technology Magazine, 9, 72-79. https://doi.org/10.1109/MVT.2014.2333763.

Devlin, J., Chang, M.-W., Lee, K., \& Toutanova, K. (2018). BERT: Pre-training of deep bidirectional transformers for language understanding. arXiv: 1810.04805 [cs].

Fonner, K. L., \& Stache, L. C. (2012). All in a day's work, at home: Teleworkers' management of micro role transitions and the work-home boundary: Teleworkers' role transitions. New Technology, Work and Employment, 27, 242-257. https://doi.org/10.1111/j.1468-005X.2012.00290.x.

Ganin, Y., Ustinova, E., \& Ajakan, H., et al. (2015). Domain-Adversarial Training of Neural Networks. arXiv: 1505.07818 [cs, stat].

Google Inc. (2019). Implementing block phone numbers. https://source.android.com/devices/tech/ connect/block-numbers. Zugegriffen am 13.09.2019.

Grießhaber, D., Vu, N. T., \& Maucher, J. (2018). Low-resource text classification using domainadversarial learning. arXiv: 1807.05195 [cs].

Henry, J. (1997). Psychological and physiological responses to stress: The right hemisphere and the hypothalamo-pituitary-adrenal axis, an inquiry into problems of human bonding. Acta Physiologica Scandinavica Supplementum, 640, 10-25.

Jäger, N. (2018). Hosting in Deutschland - Warum der Standort so wichtig ist. https://www.internetx. com/news/hosting-in-deutschland-warum-der-standort-so-wichtig-ist/. Zugegriffen am 29.06.2020. 
Mikolov, T., Chen, K., Corrado, G., \& Dean, J. (2013). Efficient estimation of word representations in vector space. arXiv: 1301.3781 [cs].

Olson, D., \& Delen, D. (2008). Advanced data mining techniques. https://doi.org/10.1007/9783-540-76917-0.

Olsson, F. (2009). A literature survey of active machine learning in the context of natural language processing (SICS technical report, 1). Kista: Swedish Institute of Computer Science. http://urn. kb.se/resolve?urn=urn:nbn:se:ri:diva-23510. Zugegriffen am 29.06.2020.

Sayah, S. (2013). Managing work-life boundaries with information and communication technologies: The case of independent contractors: Managing work-life boundaries with ICTs. New Technology, Work and Employment, 28, 179-196. https://doi.org/10.1111/ntwe.12016.

Schroeder, E. B., Liao, D., Chambless, L. E., et al. (2003). Hypertension, blood pressure, and heart rate variability: The atherosclerosis risk in communities (ARIC) study. Hypertension, 42, 1106-1111. https://doi.org/10.1161/01.HYP.0000100444.71069.73.

Shahin, M., Ali, B. M., \& Zhu, L. (2017). Continuous integration, delivery and deployment: A systematic review on approaches, tools, challenges and practices. IEEE Access, 5, 3909-3943. https://doi.org/10.1109/ACCESS.2017.2685629.

Stock, R. M., Entringer, T. M., \& Bieling, G. I. (2014). New directions for work-life balance research: A conceptual, qualitative approach. AMPROC, 2014, 16462. https://doi.org/10.5465/ ambpp.2014.16462abstract.

Tarafdar, M., Tu, Q., Ragu-Nathan, B. S., \& Ragu-Nathan, T. S. (2007). The impact of technostress on role stress and productivity. Journal of Management Information Systems, 24, 301-328. https://doi.org/10.2753/MIS0742-1222240109.

Togo, F., \& Takahashi, M. (2009). Heart rate variability in occupational health - A systematic review. Industrial Health, 47, 589-602. https://doi.org/10.2486/indhealth.47.589.

Yerushalmy, J. (1947). Statistical problems in assessing methods of medical diagnosis, with special reference to X-ray techniques. Public Health Reports (1896-1970), 62, 1432-1449.

Open Access Dieses Kapitel wird unter der Creative Commons Namensnennung 4.0 International Lizenz (http://creativecommons.org/licenses/by/4.0/deed.de) veröffentlicht, welche die Nutzung, Vervielfältigung, Bearbeitung, Verbreitung und Wiedergabe in jeglichem Medium und Format erlaubt, sofern Sie den/die ursprünglichen Autor(en) und die Quelle ordnungsgemäß nennen, einen Link zur Creative Commons Lizenz beifügen und angeben, ob Änderungen vorgenommen wurden.

Die in diesem Kapitel enthaltenen Bilder und sonstiges Drittmaterial unterliegen ebenfalls der genannten Creative Commons Lizenz, sofern sich aus der Abbildungslegende nichts anderes ergibt. Sofern das betreffende Material nicht unter der genannten Creative Commons Lizenz steht und die betreffende Handlung nicht nach gesetzlichen Vorschriften erlaubt ist, ist für die oben aufgeführten Weiterverwendungen des Materials die Einwilligung des jeweiligen Rechteinhabers einzuholen.

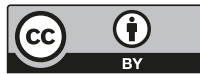

